\title{
Carbon nanotube Q-switched Yb:KLuW surface channel waveguide lasers
}

\author{
Ji Eun Bae, ${ }^{1}$ (1) Tae Gwan Park, ${ }^{1}$ Esrom Kifle, ${ }^{2}$ Xavier Mateos, ${ }^{2}$ (i) Magdalena Aguiló, ${ }^{2}$ \\ Francesc Díaz, ${ }^{2}$ Carolina Romero, ${ }^{3}$ [i] Javier Rodríguez Vázquez de Aldana, ${ }^{3}$ \\ Hansuek Lee, ${ }^{1,4}$ and Fabian Rotermund ${ }^{1, *}$ \\ ${ }^{1}$ Department of Physics, Korea Advanced Institute of Science and Technology (KAIST), 34141 Daejeon, South Korea \\ ${ }^{2}$ Física i Cristal.lografia de Materials I Nanomaterials (FiCMA-FiCNA), Universitat Rovira i Virgili (URV), E-43007 Tarragona, Spain \\ ${ }^{3}$ Aplicaciones del Láser y Fotónica, University of Salamanca, 37008 Salamanca, Spain \\ ${ }^{4}$ Graduate School of Nanoscience and Technology, Korea Advanced Institute of Science and Technology (KAIST), 34141 Daejeon, South Korea \\ ${ }^{*}$ Corresponding author: rotermund@kaist.ac.kr
}

Received 26 September 2019; revised 3 November 2019; accepted 14 November 2019; posted 18 November 2019 (Doc. ID 378820$)$; published 24 December 2019

A channel waveguide (WG) buried immediately below the surface of a $\mathrm{Yb}: \mathrm{KLuW}$ crystal is used as a laser gain medium for passive $Q$-switching by both evanescent- and directfield interactions with single-walled carbon nanotubes (SWCNTs) near $1040 \mathrm{~nm}$. The SWCNTs used as saturable absorbers (SAs) are deposited on top of the half-ring-type channel WG fabricated via femtosecond direct laser writing. The $Q$-switched WG laser delivers $88.5 \mathrm{~ns}$ pulses at a $1.16 \mathrm{MHz}$ repetition rate with a maximum average output power of $680 \mathrm{~mW}$. For the two different interaction schemes with SWCNT-SAs, the pulse characteristics, depending on the output coupling ratio and absorbed pump power, are experimentally investigated and compared to the results of theoretical analyses of the SA $Q$-switched operation. () 2019 Optical Society of America

https://doi.org/10.1364/OL.45.000216

Efficient pulsed lasers in compact cavity designs provide considerable potential for practical applications, including metrology, spectroscopy, and microscopy [1-5]. In particular, chip-size coherent sources can be usefully applied to integrated photonics. For the $1 \mu \mathrm{m}$ spectral region, ytterbium ions $\left(\mathrm{Yb}^{3+}\right)$ have been actively used as an active dopant because of their potential for realizing diode-pumped high-power lasers and ultrashort pulse generation in mode-locked lasers. Unlike glassbased gain media, $\mathrm{Yb}$-doped crystals or ceramic, particularly $\mathrm{Yb}$-doped monoclinic double tungstates such as $\mathrm{KLu}\left(\mathrm{WO}_{4}\right)_{2}$ $(\mathrm{KLuW}), \mathrm{KGd}\left(\mathrm{WO}_{4}\right)_{2}$, and $\mathrm{KY}\left(\mathrm{WO}_{4}\right)_{2}$, show excellent mechanical and thermal properties, broad and relatively high absorption/emission cross sections, and extremely low quantum defects that make them beneficial for utilization in efficient lasers.

Recently, waveguide (WG) structures have emerged as a great candidate for miniaturizing lasers. They support high intracavity pump intensities with excellent overlap between the pump and laser modes owing to well-configured guiding of the fundamental mode and, consequently, lead to low lasing thresholds and high efficiencies. To fabricate channel WGs in transparent materials, the femtosecond direct laser writing (fs-DLW) was recently suggested to be among the most reliable techniques [6,7]. Such channel WGs allow delivery of a circular single-mode output after propagating the WGs, enabling efficient laser operation.

Passive $Q$-switching utilizing a saturable absorber (SA) is a well-established method for generating nanosecond pulses in solid-state lasers. Low-dimensional materials, including graphene [8], carbon nanotubes (CNTs), topological insulators [9], and transition metal dichalcogenides (TMDs) [10] have recently become promising $S A s$ for miniaturized ultra-compact lasers $[11,12]$. They can be used for efficient pulsed operation based on direct-and evanescent-field interaction and allow monolithic integration in the cavity, while semiconductor SA mirrors and crystal-based SAs are mostly integrated between the laser gain medium and mirrors [13-15]. Regarding nanocarbons, single-walled carbon nanotubes (SWCNTs) and graphene show unique linear and nonlinear optical properties, including an intrinsic ultrafast response, large third-order nonlinearity, broadband nonlinear absorption, and flexible applicability for integration. The relatively simple, cost-effective, and controllable fabrication process of such SAs provides additional advantages for diverse forms of compact pulsed lasers.

Compact cavity utilizing $\mathrm{Yb}$-doped gain materials have been recently demonstrated in various configurations. Based on a bulk crystal without WG structures, microchip lasers have been studied because of their low losses and insensitivity to alignment, enabling higher laser efficiency and output power. The $Q$-switched Yb-doped microchip lasers have been demonstrated using Cr:YAG, TMDs, and graphene placed between the microchip and the output coupler (OC) only based on direct-field coupling [15-18].

$\mathrm{Yb}$-doped planar and channel WGs as gain media have also been investigated for the pulsed laser operation near $1 \mu \mathrm{m}$ $[12,13,19-22]$. For the evanescent-field interaction, Yb-doped 
(a)
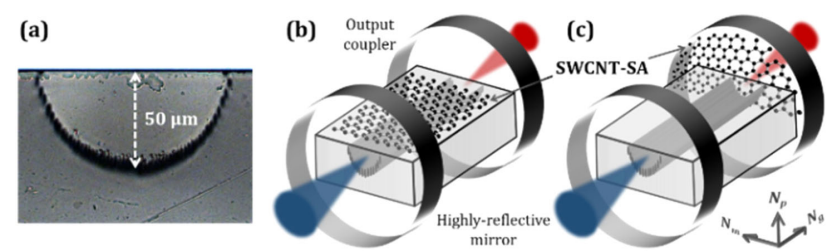

Fig. 1. (a) Optical microscope image of the surface-buried channel WG with a vertical width of $50 \mu \mathrm{m}$ and a schematic of the $Q$-switched $\mathrm{Yb}: \mathrm{KLuW}$ channel WG lasers based on (b) evanescent-and (c) directfield interaction with SWCNT-SA.

planar WG lasers are $Q$-switched using graphene and SWCNTs [12,19-21]. However, the channel WGs have the potential for improved laser efficiency, stability, and circular single-mode operation compared to that of planar WGs. Here we report diode-pumped SWCNT-Q-switched Yb:KLuW channel WG lasers based on direct-and evanescent-field interaction. To the best of our knowledge, this is the first demonstration of $Q$-switched $\mathrm{Yb}$-doped channel WG lasers based on interaction between SWCNTs and the evanescent field. SWCNTs are deposited on the top surface of the channel WG for evanescentfield coupling and the OCs for a reflective type. The $Q$-switched laser cavity consists of two different light interaction types using a $\mathrm{Yb}: \mathrm{KLuW}$ WG and laser mirrors (a highly reflective incoupling mirror and the OCs) that are attached parallel to both end facets of the WG. The $Q$-switched operation characteristics with different OC ratios and pump powers are investigated. Subsequently, the experimental results are compared to the theoretically analyzed results.

Depressed-index surface channel WGs were fabricated in a 5 at.\% $\mathrm{Yb}^{3+}$-doped $\mathrm{KLuW}$ crystal via fs-DLW. The active crystal was grown using the top-seeded-solution growth slowcooling method with $\mathrm{K}_{2} \mathrm{~W}_{2} \mathrm{O}_{7}$ as a solvent and a [010]-oriented seed [23]. Yb:KLuW is monoclinic (space group $\mathrm{C}_{2 h}^{6}-\mathrm{C} 2 / \mathrm{c}$ ) and optically biaxial. The crystal was oriented along the $N_{g}$ axis of the optical indicatrix because it provides access to the high-gain $E \| N_{m}$ polarization. The sample length was $2.88 \mathrm{~mm}$. Its aperture was $2.93\left(N_{m}\right) \times 3.05\left(N_{p}\right) \mathrm{mm}^{2}$, and both $N_{m} \times N_{p}$ faces were polished to laser-grade quality and remained uncoated. For the inscription, a Ti:sapphire regenerative amplifier emitting $120 \mathrm{fs}$ pulses at $795 \mathrm{~nm}$ at a repetition rate of $1 \mathrm{kHz}$ was used. The laser beam was focused on the crystal using a $40 \times$ microscope objective (NA. $=0.65)$. The incident pulse energy was adjusted to $65 \mathrm{~nJ}$ after the focusing optics. The crystal was scanned at $400 \mu \mathrm{m} / \mathrm{s}$ along its $N_{g}$ axis, producing damage tracks. The WG consisted of a semicircular undamaged core surrounded by a half-ring of damage tracks with a radius of $50 \mu \mathrm{m}$. The optical microscope image of the WG is shown in Fig. 1(a). The lateral separation between adjacent tracks was $2 \mu \mathrm{m}$.

The basic material used for the fabrication of SAs is arcdischarged SWCNTs (Meijo Nano Carbon Co., Ltd). The SWCNT powders, which show broadband absorption within the $1 \mu \mathrm{m}$ spectral range which corresponds to the $\mathrm{E}_{22}$ interband transitions of SWCNTs, are dissolved in 1,2-dichlorobenzene (o-DCB) with surfactant at desired concentrations. They are agitated in an ultrasonic bath. To remove bundles and impurities, the solution is centrifuged for about $20 \mathrm{~min}$. The well-dispersed SWCNT solution is then mixed with a separately prepared polymethyl methacrylate (PMMA) (Polymer Source, Inc.) solution $(100 \mathrm{mg} / \mathrm{mL}$ in o-DCB $)$.
For evanescent-field interaction with the guided beam, the SWCNT/PMMA composite solution with a SWCNT concentration of $0.5 \mathrm{mg} / \mathrm{mL}$ is spin-coated on the top surface of the $\mathrm{Yb}: \mathrm{KLuW}$ crystal containing a surface channel WG. The entire area of the top surface is coated. The front and end facet of the WG are protected during the deposition and finally cleaned. In addition, all OCs used in this Letter to study the direct-field interaction with laser beams are spin-coated under the same condition using the solution with a SWCNT concentration of $0.15 \mathrm{mg} / \mathrm{mL}$. The nonlinear optical characteristics are investigated at $1030 \mathrm{~nm}$ using SWCNT-coated $1 \mathrm{~mm}$ thick fused silica quartz. The SWCNT-SA shows a modulation depth of $0.11 \%$ and nonsaturable losses of $0.79 \%$ with a saturation fluence of $49 \mu \mathrm{J} / \mathrm{cm}^{2}$. The measured time-resolved pump-probe response can be fitted via a bi-exponential function, delivering a fast component of $251 \mathrm{fs}$ and a slow decay of $1.9 \mathrm{ps}$.

The $Q$-switched WG laser setups with two different interaction schemes employing the SWCNT-SA are shown in Figs. 1(b) and 1(c). A tapered amplifier diode laser system (TA pro, Toptica Photonics, Inc.) operating at $981.6 \mathrm{~nm}$ is used as a pump source. The pump beam passes through a half-wave plate, a polarizer, and a half-wave plate to control the incident pump power and polarization. The linearly polarized $\left(E \| N_{m}\right)$ pump beam is then focused on the WG front facet using a planoconvex lens through an incoupling mirror. This mirror, which simultaneously serves as the end mirror, is highly transparent at the pump wavelength and highly reflective at the laser wavelength. The $\mathrm{Yb}: \mathrm{KLuW}$ crystal containing the surface channel WG is mounted on the multi-axis stage without additional cooling. An OC is attached to the other end facet of the WG. The output laser beam is then analyzed after passing through a long-pass filter for filtering out the residual pump beam.

We first measured the continuous-wave (cw) performance without integration of the SWCNT-SAs. Achieved output powers versus absorbed pump powers with different $\mathrm{OC}$ ratios are shown in Fig. 2(a) with the measured beam profile at maximum output power for $T_{\mathrm{OC}}=15 \%$ (inset). The absorbed pump power is estimated by considering the Fresnel losses at the WG facets and the absorption under the lasing condition. The laser emission begins from an absorbed pump power of less than $\sim 491 \mathrm{~mW}$ in all cases. The slope efficiencies are shown to be $66 \%, 70 \%, 63 \%$, and $63 \%$ for $T_{\mathrm{OC}}=7.5,10$, 15 , and $20 \%$, respectively. The maximum $\mathrm{cw}$ output power amounted to $770 \mathrm{~mW}$ with $T_{\mathrm{OC}}=15 \%$. The propagation loss within the WG was estimated from a modified Caird analysis using $T_{\mathrm{OC}}=1$ to $20 \%$ [24]. The resultant propagation loss amounts to $0.61 \pm 0.11 \mathrm{~dB} / \mathrm{cm}$. This result indicates a highquality channel WG leading to efficient operation of the surface channel WG lasers.

The $Q$-switched operation of the $\mathrm{Yb}: \mathrm{KLuW}$ channel WG laser is achieved using SWCNT-SAs coated on top of the surface WG or on the OC. No special alignment is required for achieving the $Q$-switched operation. The measured input-output dependences for each case are plotted in Figs. 2(b) and 2(c). For $Q$-switching via the evanescent-field interaction, a maximum output power of $680 \mathrm{~mW}$ is generated with $\eta=61 \%$ at $T_{\mathrm{OC}}=15 \%$. Considering the output power observed in the cw mode, the $Q$-switching conversion from the cw operation is $90.7 \%$. The very high ratio reflects a low SA insertion loss, which leads to a highly efficient $Q$-switched operation. However, $Q$-switching via direct-field interaction provides a 

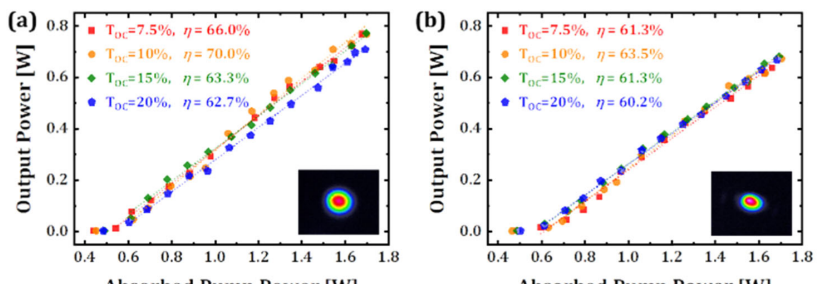

Absorbed Pump Power [W]

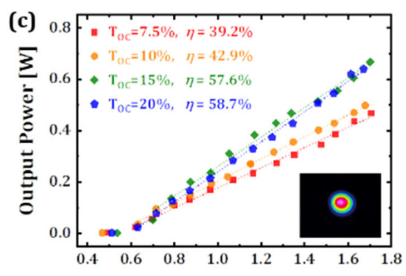

Absorbed Pump Power [W]

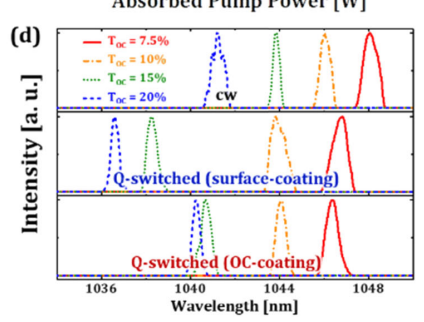

Fig. 2. Measured output power dependences in (a) cw and $Q$-switched operation based on (b) evanescent- and (c) directfield interaction (inset: beam profiles at a maximum power with $T_{\mathrm{OC}}=15 \%$ ). (d) Laser emission spectra measured in cw (top), Qswitched operation based on evanescent- (middle), and direct-field interaction (bottom).

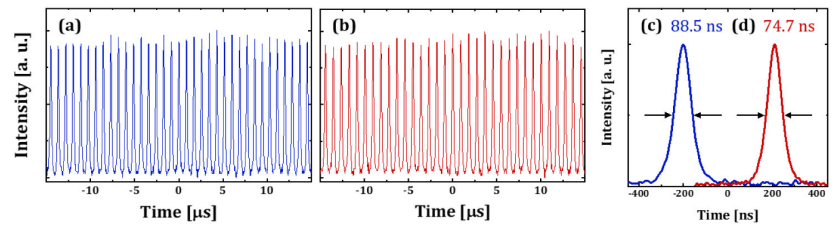

Fig. 3. $Q$-switched pulse train and the shortest pulse measured at the maximum output power with $T_{\mathrm{OC}}=15 \%$ for (a), (c) evanescentand (b), (d) direct-field interaction.

maximum power of $666 \mathrm{~mW}$ with $\eta=58 \%$ at $T_{\mathrm{OC}}=15 \%$, and the $Q$-switching conversion is $86.2 \%$. The measured $Q$-switched beam profiles at the maximum pump powers are shown in the inset of Figs. 2(b) and 2(c), and show well-defined fundamental modes in both cases. The stable $Q$-switched operation results in a pulse intensity fluctuation of $<10 \%$, as shown in Figs. 3(a) and 3(b). The recorded laser spectra of each interaction type are shown in Fig. 2(d). Because of the broadband gain of the $\mathrm{Yb}: \mathrm{KLuW}$ crystal, the spectra can be tuned near $1040 \mathrm{~nm}$. A blueshift of the laser wavelength with higher OC ratios is observed. This result is mainly attributed to increased cavity losses inducing a larger gain cross section to compensate for the losses, thus increasing the inversion ratio [23]. Note that the polarization states of both $\mathrm{cw}$ and $Q$-switched lasers are the same as those of the pump polarization $E \| N_{m}$.

The $Q$-switched pulse characteristics for both evanescentand direct-field interaction schemes are measured depending on the absorbed pump power. The pulse repetition rate $\left(f_{\text {rep }}\right)$ and pulse duration (full-width at half-maximum $\left(\Delta \tau_{p}\right)$ are directly obtained from the oscilloscope trace $(1 \mathrm{GHz}$ Tektronix, TDS784D) using a fast InGaAs photodiode. The peak power $P_{\text {peak }}$ is calculated from the measured values as $P_{\text {peak }}=P_{\text {out }} /\left(f_{\text {rep }} \cdot \Delta \tau_{p}\right)$. The results are obtained with all OCs; the case of $T_{\mathrm{OC}}=15 \%$ is summarized in Fig. 4 .

For the direct-field interaction, the pulse duration decreases from 143 to $74.7 \mathrm{~ns}$ with increasing pump power when the
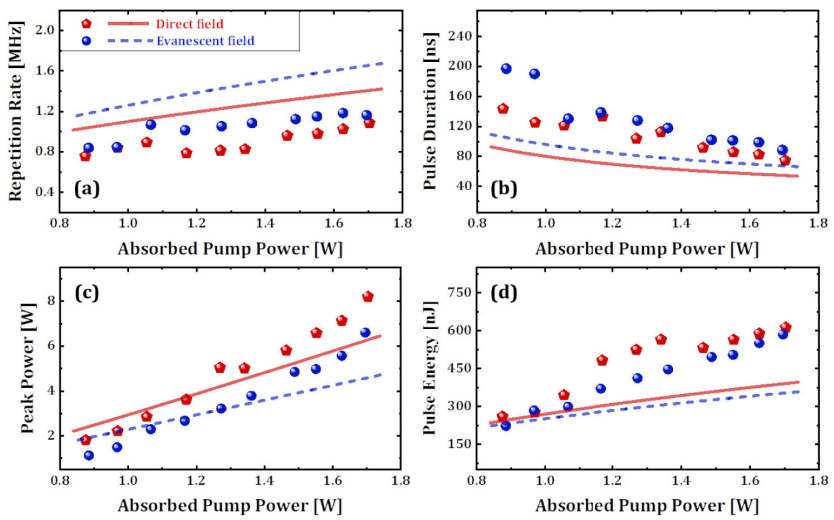

Fig. 4. $Q$-switched laser properties with $T_{\mathrm{OC}}=15 \%$ for two interaction types: (a) pulse repetition rate, (b) pulse duration, (c) pulse peak power, and (d) pulse energy (dots, experiments; lines, modeling).

repetition rate is increased from 0.758 to $1.09 \mathrm{MHz}$. The $Q$ switched pulse train and pulse width at the maximum output power are shown in Figs. 3(b) and 3(d). The maximum peak power of $8.21 \mathrm{~W}$ and the corresponding pulse energy of $613 \mathrm{~nJ}$ are obtained at the maximum pump power. Subsequently, the experimental results are compared to the results from theoretical analyses. Note that the theoretical modeling of the $Q$-switching is based on the numerical solution of the rate equations for a quasi-three-level gain medium and a fast SA having fluence-dependent nonlinear absorption [14,16,25].

The results of the pump-power-dependent theoretical analyses are also plotted in Fig. 4 as solid lines for the direct-field interaction. For theoretical modeling, the nonlinear absorption parameters of the SWCNT-SA measured in a SWCNT-coated $1 \mathrm{~mm}$ thick fused silica quartz at $1030 \mathrm{~nm}$ are used. The tendency and value of the pulse characteristics achieved during the experiment are fairly comparable to the theoretical results. However, the acceptable discrepancy might have additionally originated from practical issues, including insufficient absorption of the pump power in the WG and additional losses, including scattering loss from the SAs, as well as the WG. In addition, the higher values in peak power and pulse energy achieved in the experiment compared to the theoretical modeling are attributed to the confined geometry of our channel WG structure that results in more efficient laser operation.

For the indirect interaction, the shortest pulse duration achieved is $88.5 \mathrm{~ns}$ at a maximum pump power, accompanying an increase in the repetition rate from 0.84 to $1.16 \mathrm{MHz}$, as shown in Figs. 3(a) and 3(c). The maximum peak power is $6.61 \mathrm{~W}$, and the corresponding pulse energy is $585 \mathrm{~nJ}$. For evanescent-field interaction, it is generally not a trivial task to experimentally estimate the nonlinear absorption properties of the surface-coated SA, including the interaction peak intensity and modulation depth. Thus, we built a qualitative model based on weaker nonlinear absorption and nonsaturable loss than in the case of direct-field, owing to the longer pulse duration and lower SA insertion loss achieved, as shown in Fig. 4.

In the constructed model, assuming a modulation depth of $0.01 \%$ and a nonsaturable loss of $0.25 \%$, the pulse characteristics tend to be quite similar to the experimental results for the evanescent-field case. From this result, we can expect that the 

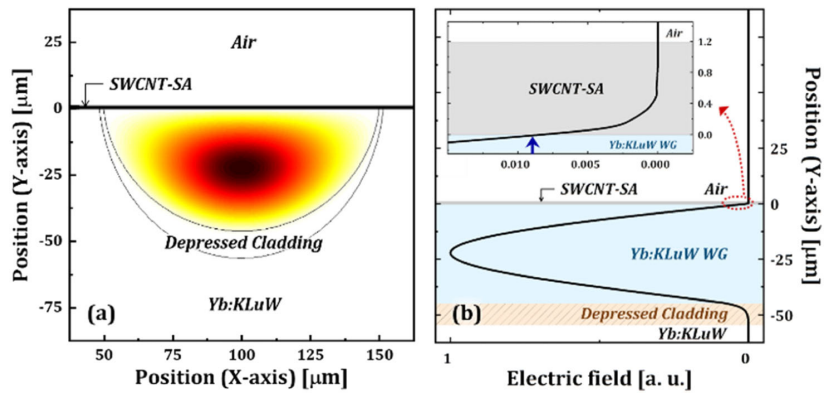

Fig. 5. (a) Calculated electric field distribution on the SWCNT SAcoated $\mathrm{Yb}: \mathrm{KLuW}$ surface channel WG and (b) its side view of the vertical cross section at the electric field maximum.

evanescent-field interaction shows a modulation of approximately $0.01 \%$ for the entire propagation of the WG which is sufficient for stable $Q$-switched operation.

The fundamental laser mode confinement within the channel WG is verified by the calculated electric field distribution, as shown in Fig. 5(a). The WG dimension is estimated from the optical microscope image (Fig. 1) and the SWCNT-SA layer thickness is $1.2 \mu \mathrm{m}$. Figure 5 (b) shows a side view of the vertical cross section at the maximum of the normalized electric field distribution in the $\mathrm{Yb}: \mathrm{KLuW}$ channel WG and SWCNTSA. The magnitude of the electric field in the SWCNT-SA is approximately $0.009 \%$ that in the $\mathrm{Yb}: \mathrm{KLuW}$ channel [inset of Fig. 5(b)]. The extracted field traveling through the WG leads to a stable $Q$-switched operation with an extremely low $Q$-switching conversion loss.

In conclusion, the $\mathrm{Yb}: \mathrm{KLuW}$ surface channel WG fabricated via fs-DLW is passively $Q$-switched by both surface-and OC-coated SWCNT-SAs. The Q-switched operation using the evanescent field is demonstrated in $\mathrm{Yb}$-doped channel WG lasers for the first time, to the best of our knowledge. The $Q$-switched WG lasers are advantageous when compared to Yb-fiber lasers $Q$-switched by evanescent-field coupling with SAs. The fiber lasers typically possess large nonsaturable losses and long cavity lengths of more than $1 \mathrm{~m}[26,27]$. Furthermore, the channel WG lasers demonstrated in this Letter show highly efficient $Q$-switched operation and a lower lasing threshold compared to that of $\mathrm{Yb}: \mathrm{KLuW}$ microchip lasers [15-18]. We can also compare our $Q$-switched lasers with $\mathrm{Yb}$-doped planar WG lasers $Q$-switched by evanescent-field interaction. Our approach provides well-defined symmetric beam modes, higher output powers, and slope efficiencies in a simplified cavity design [12,19-21]. In our case, of evanescent-field coupling, $88.5 \mathrm{~ns}$ pulses at a $1.16 \mathrm{MHz}$ repetition rate with a maximum output power of $680 \mathrm{~mW}$ are generated from a $2.88 \mathrm{~mm}$ long cavity with $T_{\mathrm{OC}}=15 \%$. The process provides a high $Q$-switching conversion from the cw mode with a very low SA insertion loss. For the direct-field interaction $74.7 \mathrm{~ns}$ pulses are achieved at a repetition rate of $1.09 \mathrm{MHz}$ with a maximum output power of $666 \mathrm{~mW}$. For both types of interaction, the characteristics of $Q$-switched pulses could be further controlled by varying the SA parameters. The experimental results compared to the theoretical results analyzed for passive $Q$-switching with a fast SA provide reasonable agreement for the pulsed characteristics.
Funding. National Research Foundation of Korea (2017R1A4A1015426, 2018H1A2A1061480); Spanish Government (FIS2017-87970-R, MAT2016-75716-C2-1-R (AEI/FEDER, UE)); Junta de Castilla y León (SA287P18); Generalitat de Catalunya (2017SGR755).

\section{REFERENCES}

1. T. M. Fortier, A. Bartels, and S. A. Diddams, Opt. Lett. 31, 1011 (2006).

2. Y. N. Billeh, M. Liu, and T. Buma, Opt. Express 18, 18519 (2010).

3. R. Aviles-Espinosa, G. Filippidis, C. Hamilton, G. Malcolm, K. J. Weingarten, T. Südmeyer, Y. Barbarin, U. Keller, S. I. C. O. Santos, D. Artigas, and P. Loza-Alvarez, Biomed. Opt. Express 2, 739 (2011).

4. M. P. Moreno and S. S. Vianna, J. Opt. Soc. Am. B 28, 2066 (2011).

5. S. Pekarek, T. Südmeyer, S. Lecomte, S. Kundermann, J. M. Dudley, and U. Keller, Opt. Express 19, 16491 (2011).

6. R. R. Gattass and E. Mazur, Nat. Photonics 2, 219 (2008).

7. F. Chen and J. R. V. de Aldana, Laser Photonics Rev. 8, 251 (2014).

8. H. Yu, X. Chen, H. Zhang, X. Xu, X. Hu, Z. Wang, J. Wang, S. Zhuang, and M. Jiang, ACS Nano 4, 7582 (2010).

9. H. Yu, H. Zhang, Y. Wang, C. Zhao, B. Wang, S. Wen, H. Zhang, and J. Wang, Laser Photonics Rev. 7, L77 (2013).

10. S. Wang, H. Yu, H. Zhang, A. Wang, M. Zhao, Y. Chen, L. Mei, and J. Wang, Adv. Mater. 26, 3538 (2014).

11. Z. Li, Y. X. Zhang, C. Cheng, H. H. Yu, and F. Chen, Opt. Express 26, 11321 (2018).

12. J. W. Kim, S. Y. Choi, J. E. Bae, M. H. Kim, Y. U. Jeong, E. Kifle, X. Mateos, M. Aguilo, F. Díaz, U. Griebner, V. Petrov, G. H. Kim, and F. Rotermund, Opt. Express 27, 1488 (2019).

13. A. A. Lagatsky, A. Choudhary, P. Kannan, D. P. Shepherd, W. Sibbett, and C. T. Brown, Opt. Express 21, 19608 (2013).

14. R. Lan, P. Loiko, X. Mateos, Y. Wang, J. Li, Y. Pan, S. Y. Choi, M. H. Kim, F. Rotermund, A. Yasukevich, K. Yumashev, U. Griebner, and V. Petrov, Appl. Opt. 55, 4877 (2016).

15. P. Loiko, J. M. Serres, X. Mateos, K. Yumashev, A. Yasukevich, V. Petrov, U. Griebner, M. Aguilo, and F. Díaz, Opt. Lett. 41, 2620 (2016).

16. P. A. Loiko, J. M. Serres, X. Mateos, J. Liu, H. Zhang, A. S. Yasukevich, K. V. Yumashev, V. Petrov, U. Griebner, M. Aguilo, and F. Díaz, Appl. Phys. B 122, 105 (2016).

17. K. Tian, X. Dou, H. Yi, W. Han, H. Xu, and J. Liu, Laser Phys. 29, 015805 (2019).

18. K. Tian, Y. H. Li, J. N. Yang, X. D. Dou, H. H. Xu, W. J. Han, and J. H. Liu, Opt. Comm. 436, 42 (2019).

19. A. Choudhary, S. J. Beecher, S. Dhingra, B. D’Urso, T. L. Parsonage, J. A. Grant-Jacob, P. Hua, J. I. Mackenzie, R. W. Eason, and D. P. Shepherd, Opt. Lett. 40, 1912 (2015).

20. J. W. Kim, S. Y. Choi, S. Aravazhi, M. Pollnau, U. Griebner, V. Petrov, S. Bae, K. J. Ahn, D. I. Yeom, and F. Rotermund, AIP Adv. 5, 017110 (2015).

21. L. Ma, Y. Tan, S. Wang, S. Akhmadaliev, S. Zhou, H. Yu, H. Zhang, and F. Chen, J. Lightwave Technol. 35, 2642 (2017).

22. S. Y. Choi, T. Calmano, F. Rotermund, and C. Kränkel, Opt. Express 26, 5140 (2018).

23. V. Petrov, M. C. Pujol, X. Mateos, O. Silvestre, S. Rivier, M. Aguilo, R. M. Sole, J. H. Liu, U. Griebner, and F. Díaz, Laser Photonics Rev. 1, 179 (2007).

24. J. A. Caird, S. A. Payne, P. R. Staver, A. J. Ramponi, L. L. Chase, and W. F. Krupke, IEEE J. Quantum Electron. 24, 1077 (1988).

25. A. S. Yasukevich, P. Loiko, N. V. Gusakova, J. M. Serres, X. Mateos, K. V. Yumashev, N. V. Kuleshov, V. Petrov, U. Griebner, M. Aguilo, and F. Díaz, Opt. Commun. 389, 15 (2017).

26. J. Lee, J. Koo, C. Chi, and J. H. Lee, J. Opt. 16, 085203 (2014).

27. J. Bogusławski, M. Kowalczyk, P. Iwanowski, A. Hruban, R. Diduszko, K. Piotrowski, K. Dybko, T. Wojciechowski, M. Aleszkiewicz, and J. Sotor, Sci. Rep. 7, 7428 (2017). 